\title{
Surfaces with a canonical principal direction and prescribed mean curvature
}

\author{
Rafael López ${ }^{1}$ - Gabriel Ruiz-Hernández ${ }^{2}$
}

Received: 16 April 2018 / Accepted: 16 January 2019 / Published online: 31 January 2019

(c) Fondazione Annali di Matematica Pura ed Applicata and Springer-Verlag GmbH Germany, part of Springer Nature 2019

\begin{abstract}
A surface in Euclidean space has a canonical principal direction with respect to a fixed direction $\mathbf{d}$ if its tangent part $\mathbf{d}^{\top}$ is a principal direction along the surface. In this paper, we classify all such surfaces with prescribed mean curvature given as an affine function of one of the following three functions: the height function, the angle function and the support function.
\end{abstract}

Keywords Principal direction · Mean curvature · Capillary surface · Self-shrinker · Translating soliton

\section{Mathematics Subject Classification 53B25}

\section{Introduction and statement of the result}

A constant angle surface in the three-dimensional Euclidean space $\mathbb{R}^{3}$ is an orientable surface whose Gauss map makes a constant angle with a fixed vector direction d ( [1]). In particular, the projection of $\mathbf{d}$ on the tangent plane of the surface is a principal direction and the corresponding principal curvature is 0 . Dillen et al. generalized, in [4], the notion of the constant angle surfaces to those surfaces where the tangent projection of $\mathbf{d}$ is a principal direction without necessarily corresponding with zero principal curvature. See also [9] for the case of hypersurfaces.

R. López: Partially supported by MEC-FEDER Grant no. MTM2017-89677-P

G. Ruiz-Hernández: This work was partially supported by UNAM-PAPIIT IN115017.

Gabriel Ruiz-Hernández gruiz@matem.unam.mx

Rafael López

rcamino@ugr.es

1 Departamento de Geometría y Topología, Instituto de Matemáticas (IEMath-GR), Universidad de Granada, 18071 Granada, Spain

2 Instituto de Matemáticas, Unidad Juriquilla, Universidad Nacional Autónoma de México, 76230 Querétaro, México 
Definition 1.1 Let $\mathbf{d} \in \mathbb{R}^{3}$ be a unitary vector. We say that a surface $M$ in $\mathbb{R}^{3}$ has a canonical principal direction with respect to $\mathbf{d}$ if the tangent part $\mathbf{d}^{\top}$ along $M$ is a principal direction.

We abbreviate by saying a CPD surface where the direction $\mathbf{d}$ is understood. The study of CPD surfaces in different ambient spaces has been of interest recently: See $[4,5,8,17]$.

The purpose of this paper is to classify all CPD surfaces whose mean curvature is a linear function of the next three functions on $M$ :

$$
\begin{aligned}
\text { height function } & f(p)=\langle p, \mathbf{v}\rangle, \\
\text { angle function } & f(p)=\langle N(p), \mathbf{v}\rangle, \\
\text { support function } & f(p)=\langle N(p), p\rangle .
\end{aligned}
$$

Here, $\mathbf{v} \in \mathbb{R}^{3}$ is a fixed unit vector and $N$ is the Gauss map of $M$. The interest for these three types of mean curvatures is the following:

1. Height function. A surface $M$ whose mean curvature $H$ satisfies

$$
H(p)=\lambda\langle p, \mathbf{v}\rangle+\mu, \lambda, \mu \in \mathbb{R},
$$

is a model of a liquid drop where the direction of the gravity is indicated by the vector $\mathbf{v}$, the constant $\lambda$ depends only on the physical properties of the drop and $\mu$ is a volume constraint: See [7] as a general reference. A surface whose mean curvature satisfies (1) is called a capillary surface.

2. Angle function. A surface $M$ whose mean curvature $H$ satisfies

$$
H(p)=\lambda\langle N(p), \mathbf{v}\rangle+\mu, \lambda, \mu \in \mathbb{R},
$$

is called a $\lambda$-translating soliton. This notion generalizes the translating soliton $(\mu=0)$. Translating solitons appear in the theory of the mean curvature flow being the equation of the limit flow by a proper blowup procedure near type II singular points $([12,13,20])$.

3. Support function. A surface $M$ whose mean curvature $H$ satisfies

$$
H(p)=\lambda\langle N(p), p\rangle+\mu, \lambda, \mu \in \mathbb{R},
$$

is called a $\lambda$-shrinker surface. This generalizes the concept of self-expander $(\lambda>0, \mu=$ $0)$ and self-shrinker $(\lambda<0, \mu=0)$. Both types of surfaces appear in the context of self-similar solutions to the mean curvature flow as models of the flow behavior near a singularity, where $M$ does not change the shape but is contracted (self-shrinkers) or dilated (self-expanders) by the flow: See $[6,11,13]$.

We observe that if $\lambda=0$ in the three above equations, then the mean curvature $H$ is constant on the surface. This particular case was treated previously in [9] (see Proposition 2.2 below).

Although in the literature, Eqs. (2) and (3) have been studied when $\mu=0$, the case $\mu \neq 0$ makes sense in the context of manifolds with density $[10,16]$. Indeed, consider $\mathbb{R}^{3}$ with a positive smooth density function $e^{\phi}, \phi \in C^{\infty}\left(\mathbb{R}^{3}\right)$, which serves as a weight for the volume and the surface area. For a variation $\left\{M_{t}: t \in(-\epsilon, \epsilon)\right\}$ of $M$, let $A_{\phi}(t)$ and $V_{\phi}(t)$ be the weighted area and the enclosed weighted volume of $M_{t}$, respectively. Then, the first variation of $A_{\phi}(t)$ and $V_{\phi}(t)$ is

$$
A_{\phi}^{\prime}(0)=-2 \int_{M} H_{\phi} u \mathrm{~d} A_{\phi}, \quad V_{\phi}^{\prime}(0)=\int_{M} u \mathrm{~d} A_{\phi},
$$

where $u$ is the normal component of the variation and $H_{\phi}=H-\langle\nabla \phi, N\rangle / 2$ is called the weighted mean curvature [19]. Then, it is immediate that $M$ is a critical point of $A_{\phi}$ 
for a given weighted volume if and only if $H_{\phi}=\mu$ is a constant function. Equations (2) and (3) appear with suitable choices of the density function $\phi$; namely, for Eq. (2), we take $\phi(p)=2 \lambda\langle p, \mathbf{v}\rangle$, and for Eq. (3), the function if $\phi(p)=\lambda|p|^{2}$.

Surfaces with a canonical principal direction have suitable parametrization. It was proved in [9] that a CPD surface with respect to $\mathbf{d}$ admits the following parametrization

$$
X(s, t)=\gamma(s)+(f(t) \eta(s)+g(t) \mathbf{d}),
$$

where $\gamma=\gamma(s), s \in I \subset \mathbb{R}$ is a curve parametrized by the arc length and contained in a plane orthogonal to $\mathbf{d}, \eta=\eta(s)$ is a unit vector field along $\gamma$ and orthogonal to both $\gamma$ and $\mathbf{d}$. Finally, $f=f(t), g=g(t), t \in J \subset \mathbb{R}$, are smooth functions that satisfy $f^{\prime}(t)^{2}+g^{\prime}(t)^{2}=1$ for all $t \in J$. The curve $\gamma$ is named the directrix of $M$, and the curve $(f(t), g(t))$ is the profile curve of $M$. As a consequence, we may take $\eta$ to be the principal normal vector to $\gamma$ and thus $\gamma^{\prime \prime}(s)=\kappa(s) \eta(s)$, where $\kappa$ is the curvature of $\gamma$.

Once introduced the above notation, we give the main result of this paper.

Theorem 1.1 Let $M$ be a CPD surface parametrized as (4). If $M$ satisfies (1), (2) or (3), then $M$ is one of the following surfaces:

1. A cylindrical surface where the base curve is $\alpha(t)=f(t) \eta+g(t) \mathbf{d}, \eta$ is a constant vector, the rulings are orthogonal to $\mathbf{d}$ and $\alpha$ satisfies the one-dimensional case of (1), (2) $\operatorname{or}(3)$.

2. A surface of revolution whose generating curve is $\alpha$ and the rotational axis is parallel to d. The curve $\alpha$ satisfies a specific second-order ODE depending on condition (1), (2) or (3).

3. A cylindrical surface where the base curve is $\gamma_{a}(s)=\gamma(s)+a \eta(s), a \in \mathbb{R}, \gamma_{a}$ satisfies the one-dimensional case of (1), (2) or (3) and the rulings are parallel to $\mathbf{d}$.

By a cylindrical surface, we mean a ruled surface where all the rulings are parallel. Then, the base curve of the surface is contained in a plane orthogonal to the rulings. In Theorem 1.1, items (1) and (3), the base curve of the cylindrical surface satisfies that its curvature is a linear function of the height, angle or support function depending on the case. In such a case, we will say then that the curve satisfies the one-dimensional case of (1), (2) or (3).

The proof of Theorem 1.1 is separated in each one of the three equations: See Sects. 3, 4 and 5 , respectively.

\section{Preliminaries}

In this section, we give an expression of the mean curvature $H$ for a CPD surface and we classify the surfaces with constant mean curvature. Firstly, we need to give some observations about parametrization (4) in the following proposition.

Proposition 2.1 Let $M$ be a CPD surface parametrized by (4).

1. We have $1-f(t) \kappa(s) \neq 0$ for all $s \in I, t \in J$.

2. If the function $g$ is constant, then $M$ is a plane orthogonal to $\mathbf{d}$.

3. If $\gamma$ is a straight line, then $M$ is a cylindrical surface whose rulings are orthogonal to $\mathbf{d}$.

4. If $\gamma$ is a circle, then $M$ is a surface of revolution whose rotational axis is parallel to $\mathbf{d}$.

Proof 1 . Since $\partial_{s} X=(1-\kappa(s) f(t)) \gamma^{\prime}(s) \neq 0$ by regularity of $M$, we conclude that $1-\kappa(s) f(t) \neq 0$. 
2. If $g(t)=a$ for all $s \in I$, then $f(t)=\epsilon t+b$, with $b \in \mathbb{R}$ and $\epsilon \in\{-1,1\}$. Then, (4) gives

$$
X(s, t)=\gamma(s)+(\epsilon t+b) \eta(s)+a \mathbf{d}=(\gamma(s)+a \mathbf{d})+(\epsilon t+b) \eta(s) .
$$

It follows that $\gamma(s)+a \mathbf{d}$ is a planar curve and the curves $t \mapsto(\epsilon t+b) \eta(s)$ are segments of straight lines coplanar to $\gamma$. This proves that $M$ is a plane orthogonal to d.

3. If $\gamma$ is a straight line, then $\eta$ is a unit constant vector and from (4), the surface $M$ is a cylindrical surface whose base curve is $\alpha(t)=f(t) \eta+g(t) \mathbf{d}$ and the rulings are orthogonal to d.

4. After a change of coordinates, we suppose $\gamma(s)=(r \cos (s), r \sin (s), 0)$ and $\mathbf{d}=$ $(0,0,1)$. By (4), we find

$$
X(s, t)=(r(1-f(t)) \cos (s), r(1-f(t)) \sin (s), g(t)),
$$

proving that $M$ is a surface of revolution about the $\mathbf{d}$-axis.

We need to have an expression of the mean curvature $H$ of a CPD surface. The following result is proved in [9].

Proposition 2.2 Let $M$ be a CPD surface. If $M$ is not a plane orthogonal to $\mathbf{d}$, then the principal curvatures $\kappa_{1}$ and $\kappa_{2}$ with respect to the Gauss map $N(s, t)=-g^{\prime}(t) \eta(s)+f^{\prime}(t) \mathbf{d}$ are

$$
\kappa_{1}(X(s, t))=\frac{f^{\prime \prime}(t)}{g^{\prime}(t)}, \quad \kappa_{2}(X(s, t))=\frac{g^{\prime}(t) \kappa(s)}{1-f(t) \kappa(s)} .
$$

The mean curvature $H$ with respect to $N$ is

$$
H(X(s, t))=\frac{1}{2}\left(\frac{f^{\prime \prime}(t)}{g^{\prime}(t)}+\frac{g^{\prime}(t) \kappa(s)}{1-f(t) \kappa(s)}\right) .
$$

In Theorem 1.1, we exclude the case $\lambda=0$, that is, that the surface has constant mean curvature. The CPD surfaces with constant mean curvature were obtained in [9, Cor. 14]. By completeness, we give its proof.

Proposition 2.3 If $M$ is a CPD surface with constant mean curvature $H$, then $M$ is a plane, a circular cylinder with axis orthogonal to $\mathbf{d}$ or a surface of revolution whose axis is parallel to $\mathbf{d}$.

Proof It is immediate that a plane orthogonal to $\mathbf{d}$ is a CPD surface with respect to $\mathbf{d}$ and with zero mean curvature. Suppose now that $M$ is not the above surface, in particular, the function $g(t)$ in (4) is not constant by item 2 of Proposition 2.1. By Proposition 2.2, we have

$$
\frac{f^{\prime \prime}(t)}{g^{\prime}(t)}+\frac{g^{\prime}(t) \kappa(s)}{1-f(t) \kappa(s)}=2 H,
$$

where the right-hand side is a constant. Differentiating with respect to $s$, we obtain

$$
\frac{g^{\prime}(t) \kappa^{\prime}(s)}{(1-f(t) \kappa(s))^{2}}=0 .
$$

Since $g^{\prime}(t) \neq 0$, we conclude $\kappa^{\prime}(s)=0$ for every $s \in I$. This means that $\gamma$ is a straight line or a circle. 
1. Case $\gamma$ is a straight line. By Proposition 2.1, the surface $M$ is a cylindrical surface whose rulings are parallel to $\gamma$. Since $H$ is constant, then $M$ is a plane parallel to $\gamma(H=0)$ or $M$ is a circular cylinder $(H \neq 0)$ whose axis is the straight line, being $\gamma$ orthogonal to d.

2. Case $\gamma$ is a circle. By Proposition 2.1 again, $M$ is a surface of revolution whose axis is parallel to d.

\section{CPD capillary surfaces}

In this section, we prove Theorem 1.1 for capillary surfaces. By the expression of $H$ in (5), Eq. (1) is written as

$$
\frac{1}{2}\left(\frac{f^{\prime \prime}(t)}{g^{\prime}(t)}+\frac{g^{\prime}(t) \kappa(s)}{1-f(t) \kappa(s)}\right)=\lambda(\langle\gamma(s), \mathbf{v}\rangle+(f(t)\langle\eta(s), \mathbf{v}\rangle+g(t)\langle\mathbf{d}, \mathbf{v}\rangle))+\mu,
$$

for all $s \in I, t \in J$. We differentiate with respect to $s$, obtaining

$$
\frac{g^{\prime}(t) \kappa^{\prime}(s)}{(1-f(t) \kappa(s))^{2}}=2 \lambda(1-f(t) \kappa(s))\left\langle\gamma^{\prime}(s), \mathbf{v}\right\rangle,
$$

where we have used $\gamma^{\prime \prime}(s)=\kappa(s) \eta(s)$. We discuss according to $\kappa$.

1. Case $\kappa^{\prime}(s)=0$ for every $s \in I$. Then, the curvature function $\kappa$ is constant. Moreover, Eq. (6) implies $\left\langle\gamma^{\prime}(s), \mathbf{v}\right\rangle=0$, that is, $\langle\gamma(s), \mathbf{v}\rangle$ is a constant function. From Proposition 2.1, we know that $M$ is a cylindrical surface whose rulings are orthogonal to $\mathbf{d}$ or $M$ is a surface of revolution whose axis is parallel to $\mathbf{d}$.

(a) Case $\kappa=0$. Then, $\eta$ is a constant vector and Eq. (1) becomes

$$
\frac{1}{2} \frac{f^{\prime \prime}(t)}{g^{\prime}(t)}=\lambda\langle f(t) \eta+g(t) \mathbf{d}, \mathbf{v}\rangle+\lambda\langle\gamma(s), \mathbf{v}\rangle+\mu .
$$

Define $\alpha(t)=f(t) \eta+g(t) \mathbf{d}$ and $\tilde{\mu}=\lambda\langle\gamma(s), \mathbf{v}\rangle+\mu$. Let us observe that $\tilde{\mu}$ is constant. On the other hand, $\alpha$ is parametrized by the arc length and its curvature $\tilde{\kappa}$ satisfies

$$
\frac{\tilde{\kappa}(t)}{2}=\lambda\langle\alpha(t), \mathbf{v}\rangle+\tilde{\mu}
$$

Consequently, the planar curve $\alpha$ satisfies the one-dimensional case of Eq. (1) as a linear combination of its height function.

(b) Case $\kappa \neq 0$. We know that $M$ is a rotational surface whose axis is parallel to $\mathbf{d}$. Since $\left\langle\gamma^{\prime}(s), \mathbf{v}\right\rangle=0$, a differentiation with respect to $s$ yields $\kappa(s)\langle\eta(s), \mathbf{v}\rangle=0$. We deduce that $\langle\eta(s), \mathbf{v}\rangle=0$, and it follows that $\mathbf{v}$ is orthogonal to both $\gamma^{\prime}$ and $\eta$. This says that $\mathbf{d}$ is parallel to $\mathbf{v}$. By Eq. (1), the generating curve $\alpha(t)=f(t) \eta+g(t) \mathbf{d}$ satisfies the ODE

$$
\frac{1}{2}\left(\frac{f^{\prime \prime}(t)}{g^{\prime}(t)}+\frac{g^{\prime}(t) \kappa}{1-f(t) \kappa}\right)=\epsilon \lambda g(t)+b
$$

where $\epsilon=\langle\mathbf{d}, \mathbf{v}\rangle= \pm 1$ and $b=\lambda\langle\gamma(s), \mathbf{v}\rangle+\mu$ is a constant. 
2. Case $\kappa^{\prime}\left(s_{0}\right) \neq 0$ at some $s_{0} \in I$. In particular, $\kappa^{\prime}(s) \neq 0$ in a subinterval $I^{\prime}$ of $I$ around $s=s_{0}$. By simplicity, we assume $I^{\prime}=I$. We now write (6) as

$$
2 \frac{\lambda\left\langle\gamma^{\prime}(s), \mathbf{v}\right\rangle}{\kappa^{\prime}(s)}=\frac{g^{\prime}(t)}{(1-f(t) \kappa(s))^{3}} .
$$

By differentiating with respect to $t$, we have

$$
g^{\prime \prime}(t)(1-f(t) \kappa(s))+3 g^{\prime}(t) f^{\prime}(t) \kappa(s)=0 .
$$

We distinguish two possibilities:

(a) Subcase $g^{\prime \prime}(t) \neq 0$ in some $t_{0} \in J$. Then, $g^{\prime \prime}(t) \neq 0$ in an interval $K \subset J$ around $t=t_{0}$. In $I \times K$, we can write (7) as

$$
\frac{1}{\kappa(s)}=f(t)-3 \frac{g^{\prime}(t) f^{\prime}(t)}{g^{\prime \prime}(t)} .
$$

Since the left-hand side depends only on $s$ and the right-hand side on $t$, then they are constant functions; in particular, $1 / \kappa(s)$ is constant, a contradiction because $\kappa^{\prime}(s) \neq$ 0 .

(b) Subcase $g^{\prime \prime}(t)=0$ for every $t$. From (7), we have $f^{\prime}(t) \kappa(s)=0$ for every $s \in I$, $t \in J$. Since $\kappa^{\prime}(s) \neq 0$, then $\kappa(s) \neq 0$ for some $s \in I$, and thus $f^{\prime}(t)=0$ for every $t \in J$. Hence, $g^{\prime}(t)=\epsilon \in\{-1,1\}, f$ is a constant function $f(t)=a, a \in \mathbb{R}$, and $g(t)=\epsilon t+b, b \in \mathbb{R}$. Parametrization (4) of $M$ becomes

$$
X(s, t)=\gamma(s)+a \eta(s)+(\epsilon t+b) \mathbf{d} .
$$

This proves that $M$ is a cylindrical surface whose base curve is the planar curve $\gamma_{a}(s)=\gamma(s)+a \eta(s)$ and the rulings are parallel to $\mathbf{d}$. Under this situation, Eq. (1) becomes

$$
\frac{\epsilon \kappa(s)}{2(1-a \kappa(s))}=\lambda\left(\left\langle\gamma_{a}(s), \mathbf{v}\right\rangle+(\epsilon t+b)\langle\mathbf{d}, \mathbf{v}\rangle\right)+\mu .
$$

The derivative with respect to $t$ gives $\lambda \epsilon\langle\mathbf{d}, \mathbf{v}\rangle=0$, and we deduce that $\mathbf{d}$ is orthogonal to v. Eq. (1) simplifies into

$$
\frac{\kappa(s)}{1-a \kappa(s)}=2 \epsilon \lambda\left\langle\gamma_{a}(s), \mathbf{v}\right\rangle+2 \epsilon \mu
$$

Finally, a simple computation gives that the curvature of $\gamma_{a}$ is $\kappa_{\gamma_{a}}=\frac{\epsilon \kappa(s)}{1-a \kappa(s)}$. Thus, from (8), we deduce that $\gamma_{a}$ satisfies the one-dimensional case of (1).

We point out that the one-dimensional case of capillary Eq. (1) and the rotational capillary surfaces are well known. For the first one, we refer [18, pp. 1130-40], also [14]. A detailed description of the rotational capillary surfaces lies in [7].

\section{CPD $\lambda$-translating solitons}

We now give the proof of Theorem 1.1 for CPD $\lambda$-translating solitons. In order to compute (2), we need to know $\langle N(p), \mathbf{v}\rangle$. By Proposition 2.2, we find

$$
\langle N(s, t), \mathbf{v}\rangle=-g^{\prime}(t)\langle\eta(s), \mathbf{v}\rangle+f^{\prime}(t)\langle\mathbf{d}, \mathbf{v}\rangle .
$$


By combining (2) and (5), we obtain

$$
\frac{f^{\prime \prime}(t)}{g^{\prime}(t)}+\frac{g^{\prime}(t) \kappa(s)}{1-f(t) \kappa(s)}=-2 \lambda g^{\prime}(t)\langle\eta(s), \mathbf{v}\rangle+2 \lambda f^{\prime}(t)\langle\mathbf{d}, \mathbf{v}\rangle+2 \mu,
$$

for all $s \in I, t \in J$. By taking the derivative with respect to $s$, and using that $g^{\prime}(t) \neq 0$, we get

$$
\frac{\kappa^{\prime}(s)}{(1-f(t) \kappa(s))^{2}}=-2 \lambda\left\langle\eta^{\prime}(s), \mathbf{v}\right\rangle=2 \lambda \kappa(s)\left\langle\gamma^{\prime}(s), \mathbf{v}\right\rangle .
$$

We differentiate this expression with respect to $t$, obtaining now

$$
\frac{2 \kappa(s) \kappa^{\prime}(s) f^{\prime}(t)}{(1-f(t) \kappa(s))^{3}}=0 .
$$

Then, $\left(\kappa^{2}(s)\right)^{\prime} f^{\prime}(t)=0$. We discuss two cases.

1. Suppose there exists $t_{0} \in J$ such that $f^{\prime}\left(t_{0}\right) \neq 0$. Then, $\kappa$ is a constant function. In such a case, by Proposition 2.1 we conclude that $M$ is either a cylindrical surface whose rulings are orthogonal to $\mathbf{d}$ or $M$ is a surface of revolution with axis parallel to $\mathbf{d}$.

(a) Case $\kappa=0$. Now $\eta$ is a constant vector. Equation (2) is now

$$
\frac{f^{\prime \prime}(t)}{g^{\prime}(t)}=-2 \lambda g^{\prime}(t)\langle\eta(s), \mathbf{v}\rangle+2 \lambda f^{\prime}(t)\langle\mathbf{d}, \mathbf{v}\rangle+2 \mu .
$$

Define the planar curve $\alpha(t)=f(t) \eta+g(t) \mathbf{d}$ is a planar curve. A computation of its principal normal vector $N_{\alpha}$ gives $N_{\alpha}(s)=-g^{\prime}(t) \eta+f^{\prime}(t) \mathbf{d}$ and its curvature is $\kappa_{\alpha}(t)=\frac{f^{\prime \prime}(t)}{g^{\prime}(t)}$. Thus, (9) is written as

$$
\kappa_{\alpha}(t)=2 \lambda\left\langle N_{\alpha}(t), \mathbf{v}\right\rangle+2 \mu,
$$

proving that $\alpha$ satisfies the one-dimensional case of (2).

(b) Case $\kappa \neq 0$. Then, $\left\langle\gamma^{\prime}(s), \mathbf{v}\right\rangle=0$, that is, $\langle\gamma(s), \mathbf{v}\rangle$ is a constant function. Moreover, taking another derivative with respect to $s$, we find $\kappa\langle\eta(s), \mathbf{v}\rangle=0$, so $\mathbf{v}$ is orthogonal to both $\gamma^{\prime}(s)$ and $\eta(s)$ which proves that $\mathbf{v}$ is parallel to $\mathbf{d}$. Now Eq. (2) becomes

$$
\frac{f^{\prime \prime}(t)}{g^{\prime}(t)}+\frac{g^{\prime}(t) \kappa}{1-f(t) \kappa}=2 \epsilon \lambda f^{\prime}(t)+2 \mu,
$$

with $\epsilon=\langle\mathbf{d}, \mathbf{v}\rangle= \pm 1$.

2. Case $f^{\prime}(t)=0$ for every $t \in J$. Then, $f$ is a constant function $f(t)=a, a \in \mathbb{R}$. From $f^{\prime}(t)^{2}+g^{\prime}(t)^{2}=1$, we deduce that $g(t)=\epsilon t+b, \epsilon \in\{-1,1\}, b \in \mathbb{R}$. The parametrization $X$ in (4) is now

$$
X(s, t)=(\gamma(s)+a \eta(s))+(\epsilon t+b) \mathbf{d} .
$$

This proves that $M$ is a cylindrical surface over the planar curve $\gamma_{a}(s)=\gamma(s)+a \eta(s)$ and the rulings are parallel to d. Now Eq. (2) becomes

$$
\frac{\kappa(s)}{1-a \kappa(s)}=-2 \lambda\langle\eta(s), \mathbf{v}\rangle+2 \mu \epsilon .
$$

Again the left-hand side of (10) is the curvature $\kappa_{\gamma_{a}}$ of $\gamma_{a}$, and consequently (10) asserts that $\gamma_{a}$ satisfies the one-dimensional case of (2).

We point out that the rotational surfaces that are $\lambda$-translating solitons are classified in [3] if $\mu=0$ and in [15] when $\mu \neq 0$. 


\section{CPD $\lambda$-shrinker surfaces}

We now give the proof of Theorem 1.1 for CPD $\lambda$-shrinker surface. From the expression of $N$ in Proposition 2.2, we have

$$
\begin{aligned}
\langle N(p), p\rangle & =\left\langle-g^{\prime}(t) \eta(s)+f^{\prime}(t) d, \gamma(s)+(f(t) \eta(s)+g(t) d)\right\rangle \\
& =-g^{\prime}(t)\langle\eta(s), \gamma(s)\rangle-f(t) g^{\prime}(t)+f^{\prime}(t) g(t) .
\end{aligned}
$$

By the expression of $H$ in (5), Eq. (3) is written as

$$
\frac{f^{\prime \prime}(t)}{g^{\prime}(t)}+\frac{g^{\prime}(t) \kappa(s)}{1-f(t) \kappa(s)}=-2 \lambda g^{\prime}(t)\langle\eta(s), \gamma(s)\rangle-2 \lambda f(t) g^{\prime}(t)+2 \lambda f^{\prime}(t) g(t)+2 \mu,
$$

for all $s \in I, t \in J$. Since $g^{\prime}(t) \neq 0$, we divide the above expression by $g^{\prime}(t)$, obtaining

$$
\frac{f^{\prime \prime}(t)}{g^{\prime}(t)^{2}}+\frac{\kappa(s)}{1-f(t) \kappa(s)}=-2 \lambda\langle\eta(s), \gamma(s)\rangle-2 \lambda f(t)+2 \lambda \frac{f^{\prime}(t) g(t)}{g^{\prime}(t)}+2 \frac{\mu}{g^{\prime}(t)} .
$$

We observe that the right-hand side in the above equation is a sum of a function depending only on $s$ and other function depending only on $t$. Thus, if we differentiate with respect to $s$ and then with respect to $t$, we obtain 0 . Therefore, looking at the left-hand side, we conclude

$$
0=\frac{2 \kappa(s) \kappa^{\prime}(s) f(t)}{(1-f(t) \kappa(s))^{3}}=\frac{\left(\kappa^{2}(s)\right)^{\prime} f(t)}{(1-f(t) \kappa(s))^{3}} .
$$

Then, $\left(\kappa^{2}(s)\right)^{\prime} f^{\prime}(t)=0$. Now the discussion follows the next two cases.

1. Suppose there exists $t_{0} \in J$ such that $f^{\prime}\left(t_{0}\right) \neq 0$. Then, $\kappa$ is a constant function. In such a case, by Proposition 2.1, we conclude that $M$ is either a cylindrical surface whose rulings are orthogonal to $\mathbf{d}$ or $M$ is a surface of revolution with axis parallel to $\mathbf{d}$. By the above equations, $\kappa$ constant implies that the function $\langle\gamma(s), \eta(s)\rangle$ is constant.

(a) Case $\kappa=0$. In particular, $\gamma^{\prime}$ and $\eta$ are constant coplanar vectors and mutually orthogonal. Define the curve $\alpha(t)=f(t) \eta+g(t)$ d. The principal normal vector of $\alpha$ is $N_{\alpha}(t)=-g^{\prime}(t) \eta+f^{\prime}(t)$ d. Equation (3) is written as

$$
\frac{f^{\prime \prime}(t)}{g^{\prime}(t)}=2 \lambda\left\langle N_{\alpha}(t), \gamma(s)+\alpha(t)\right\rangle+2 \mu .
$$

Let us observe that $\left\langle N_{\alpha}(t), \gamma(s)\right\rangle$ does not depend on $s$ and $\kappa_{\alpha}(t)=f^{\prime \prime}(t) / g^{\prime}(t)$ is the curvature of $\alpha$. Consequently, $\alpha$ satisfies the one-dimensional case of (3).

(b) Case $\kappa \neq 0$. Then, $\gamma$ is a circle. Furthermore, we have

$$
-\kappa\left\langle\gamma(s), \gamma^{\prime}(s)\right\rangle=\left\langle\gamma(s), \eta^{\prime}(s)\right\rangle=\langle\gamma(s), \eta(s)\rangle^{\prime}=0,
$$

which implies that $\left\langle\gamma(s), \gamma^{\prime}(s)\right\rangle=0$. This shows that $\gamma$ is a circle with center in the origin. Taking another derivative, we find

$$
\left\langle\gamma(s), \gamma^{\prime}(s)\right\rangle^{\prime}=\left\langle\gamma(s), \gamma^{\prime \prime}(s)\right\rangle+\left\langle\gamma^{\prime}(s), \gamma^{\prime}(s)\right\rangle=\kappa\langle\gamma(s), \eta(s)\rangle+1=0 .
$$

Therefore, $\langle\gamma(s), \eta(s)\rangle=-1 / \kappa$. Thus, $M$ is a rotational surface whose axis of revolution passes through the origin. Moreover, the generating curve satisfies

$$
\frac{f^{\prime \prime}(t)}{g^{\prime}(t)}+\frac{g^{\prime}(t) \kappa}{1-f(t) \kappa}=(2 / \kappa) \lambda g^{\prime}(t)-2 \lambda f(t) g^{\prime}(t)+2 \lambda f^{\prime}(t) g(t)+2 \mu .
$$


2. Case $f^{\prime}(t)=0$ for every $t \in J$. Then, $f$ is a constant function $f(t)=a, a \in \mathbb{R}$. From $f^{\prime}(t)^{2}+g^{\prime}(t)^{2}=1$, we deduce that $g(t)=\epsilon t+b, \epsilon \in\{-1,1\}, b \in \mathbb{R}$. From (4), the parametrization $X$ is written as

$$
X(s, t)=(\gamma(s)+a \eta(s))+(\epsilon t+b) \mathbf{d} .
$$

This proves that $M$ is a cylindrical surface over the planar curve $\gamma_{a}=\gamma(s)+a \eta(s)$ and the rulings are parallel to d. Finally, Eq. (3) becomes

$$
\frac{\kappa(s)}{1-a \kappa(s)}=-2 \lambda\langle\eta(s), \gamma(s)\rangle-2 \lambda a+2 \epsilon \mu=2 \lambda\left\langle\eta(s), \gamma_{a}(s)\right\rangle+2 \epsilon \mu .
$$

Because the curvature of $\gamma_{a}$ is $\kappa_{\gamma_{a}}=\kappa(s) /(1-a \kappa(s))$, then $\gamma_{a}$ satisfies the onedimensional case of (3).

The cylindrical shrinker surfaces are classified in [2].

Acknowledgements The second author wants to thanks the Departamento de Geometría y Topología and the Instituto de Matemáticas (IEMath-GR) of Universidad de Granada for the hospitality, facilities and inspiring ambient during this sabbatical year. He is also grateful with his institution UNAM by this opportunity of a sabbatical period supported by the program PASPA of DGAPA.

\section{References}

1. Cermelli, P., Di Scala, A.J.: Constant-angle surfaces in liquid crystals. Philos. Mag. 87, 1871-1888 (2007)

2. Chang, J.-E.: 1-dimensional solutions of the $\lambda$-self shrinkers. Geom. Dedicata 189, 97-112 (2017)

3. Clutterbuck, J., Schnürer, O., Schulze, F.: Stability of translating solutions to mean curvature flow. Calc. Var. 29, 281-293 (2007)

4. Dillen, F., Fastenakels, J., Van der Veken, J.: Surfaces in $S^{2} \times \mathbb{R}$ with a canonical principal direction. Ann. Glob. Anal. Geom. 35, 381-396 (2009)

5. Dillen, F., Munteanu, M.I., Nistor, A.: Canonical coordinates and principal directions for surfaces in $\mathbb{H}^{2} \times \mathbb{R}$. Taiwan. J. Math. 15, 2265-2289 (2011)

6. Ecker, K., Huisken, G.: Mean curvature evolution of entire graphs. Ann. Math. 130, 453-471 (1989)

7. Finn, R.: Equilibrium Capillary Surfaces. Springer, Berlin (1986)

8. Fu, Y., Nistor, A.: Constant angle property and canonical principal directions for surfaces in $M^{2}(c) \times R_{1}$. Mediterr. J. Math. 10, 1035-1049 (2013)

9. Garnica, E., Palmas, O., Ruiz-Hernández, G.: Hypersurfaces with a canonical principal direction. Differ. Geom. Appl. 30, 382-391 (2012)

10. Gromov, M.: Isoperimetry of waists and concentration of maps. Geom. Funct. Anal. 13, 178-215 (2003)

11. Huisken, G.: Flow by mean curvature convex surfaces into spheres. J. Differ. Geom. 20, 237-266 (1984)

12. Huisken, G., Sinestrari, C.: Mean curvature flow singularities for mean convex surfaces. Calc. Var. 8, 1-14 (1999)

13. Ilmanen, T.: Lectures on mean curvature flow and related equations. In: Conference on Partial Differential Equations \& Applications to Geometry. ICTP, Trieste (1995)

14. López, R.: Capillary channels in a gravitational field. Nonlinearity 20, 1573-1600 (2007)

15. López, R.: Invariant surfaces in Euclidean space with a log-linear density. Adv. Math. 339, 285-309 (2018)

16. Morgan, F.: Manifolds with density. Not. Am. Math. Soc. 52, 853-858 (2005)

17. Munteanu, M.I., Nistor, A.: Complete classification of surfaces with a canonical principal direction in the Euclidean space $E^{3}$. Cent. Eur. J. Math. 9, 378-389 (2011)

18. Pockels, F.: Kapillarität Handbuch der Physik Band I ed A Winkelmann. Leipzig (1908)

19. Rosales, C., Cañete, A., Bayle, V., Morgan, F.: On the isoperimetric problem in Euclidean space with density. Calc. Var. Partial Differ. Equ. 31, 27-46 (2008)

20. Wa, X.-J.: Convex solutions to the mean curvature flow. Ann. Math. 173, 1185-1239 (2011)

Publisher's Note Springer Nature remains neutral with regard to jurisdictional claims in published maps and institutional affiliations. 